\title{
Analysis of WIMAX Physical Layer using Spatial Diversity
}

\author{
Pavani Sanghoi \\ Student, Department of Electronics and \\ Communication Engineering \\ Lovely Professional University, Jalandhar, \\ Punjab, India
}

\author{
Lavish Kansal \\ Assistant Professor, Department of Electronics \\ and Communication Engineering \\ Lovely Professional University, Jalandhar, \\ Punjab, India
}

\begin{abstract}
IEEE 802.16 standard for Broadband Wireless Access (BWA) and its associated industry consortium, Worldwide Interoperability for Microwave Access (WiMAX) forum promise to offer high data rate over large areas to a large number of users where broadband is unavailable. The emergence of WiMAX has attracted significant interests from all the fields of wireless communications. WiMAX-MIMO systems which are created under different fading channels can be implemented to get the benefits of both WiMAX and MIMO systems. This paper analyzes different higher level modulations on WiMAX-MIMO systems with different code rates for Rayleigh channel. Spatial Diversity technique is taken into consideration for the simulation purpose. Signal-to Noise Ratio (SNR) vs Bit Error Rate (BER) analysis has been done.
\end{abstract}

\section{Keywords}

BWA, WiMAX, MIMO, SNR, BER, FEC, CC, PHY

\section{INTRODUCTION}

WiMAX standard is the commercialization of the IEEE 802.16 standard that provides high speed Broadband Wireless Access to the users. It aims to provide business and consumer wireless broadband services on the scale of the Metropolitan Area Network (MAN). WiMAX is a Wireless MAN (WAN) technology, which fits between WLANs and wireless wide area networks (WANs). It has been developed to provide cost effective, high-quality, and flexible BWA solutions using certified, compatible and interoperable equipments from different vendors. WiMAX can provide broadband services to people who could not afford wired broadband services before and cover areas where broadband services have not been available before. The distance of a WiMAX connection can be up to 30 miles $(50 \mathrm{~km})$ at data rates up to $75 \mathrm{Mbps}$ using both the unlicensed and licensed spectrums. The IEEE 802.16e air interface standard [1] is based on orthogonal frequencydivision multiplexing (OFDM), which has been regarded as an efficient way to combat the inter-symbol interference (ISI).

The first version of the IEEE 802.16 standard operates in the $10-66 \mathrm{GHz}$ frequency band and requires line-of-sight (LOS) towers called Fixed WiMAX [2]. Later the standard extended its operation through different PHY specification $2-11 \mathrm{GHz}$ frequency band enabling non line of sight (NLOS) connections called Mobile WiMAX, which require techniques that efficiently mitigate the impairment of fading and multipath [3].

MIMO technology has attracted attention in wireless communications, since it offers significant increases in data throughput and link range without additional bandwidth or transmit power. It achieves this by higher spectral efficiency and link reliability or diversity. It generally refers to the use of multiple antennas both at the transmitter and receiver to improve the performance of radio communication systems. A MIMO system takes advantage of the spatial diversity that is obtained by spatially separated antennas in a dense multipath scattering environment. MIMO systems may be implemented in a number of different ways to obtain either a diversity gain to combat signal fading or to obtain a capacity gain [4].

The paper is organized as follows: model of WiMAX PHY layer is explained in section II. A brief overview of MIMO systems is presented in Section III. MIMO Techniques are provided in section IV. WiMAX-MIMO systems are studied in section V. Results and simulations are shown in section VI. At the end conclusion is given in section VII.

\section{WIMAX MODEL FOR PHYSICAL LAYER}

The physical layer of WiMAX is quite flexible; hence the data rate performance varies based on the operating parameters. Parameters that have a significant impact on the physicallayer data rate are channel bandwidth and the modulation and coding scheme used. Other parameters, such as number of sub-channels, OFDM guard time, and oversampling rate, also have an impact. The block diagram for WiMAX system is shown in figure 1 [5]. Now we will take each block one by one in detail.

\subsection{Randomization}

Randomization introduces protection through informationtheoretic uncertainty, avoiding long sequences of consecutive ones or consecutive zeros. It is also useful for avoiding noncentred data sequenes. Data randomization is performed on each downlink and uplink burst of data. It increases the coding performance and data integrity.

\subsection{Forward Error Correction (FEC)}

The bits issued from the randomizer are then applied to the FEC encoder. FEC techniques typically use error-correcting codes that can detect with high probability the error location. These channel codes improve the bit error rate performance by adding redundant bits in the transmitted bit stream that are employed by the receiver to correct errors introduced by the channel. This reduces the signal transmit power for a given BER

\subsubsection{RS codes}

These code are mandatory codes on both the sides i.e. uplink and downlink. These are non-binary cyclic codes that add redundancy to the data that improves the probability of block errors. 


\subsubsection{Convolution codes $(C C)$}

These codes are used to correct the random errors [6]. If there are $\mathrm{k}$ bits per second input to the convolutional encoder and the output is $\mathrm{n}$ bits per second, the code rate is $\mathrm{k} / \mathrm{n}$. The redundancy is on not only the incoming $\mathrm{k}$ bits, but also several of the preceding $\mathrm{k}$ bits. Preceding $\mathrm{k}$ bits used in the encoding process is the constraint length $\mathrm{m}$ that is similar to the memory in the system.

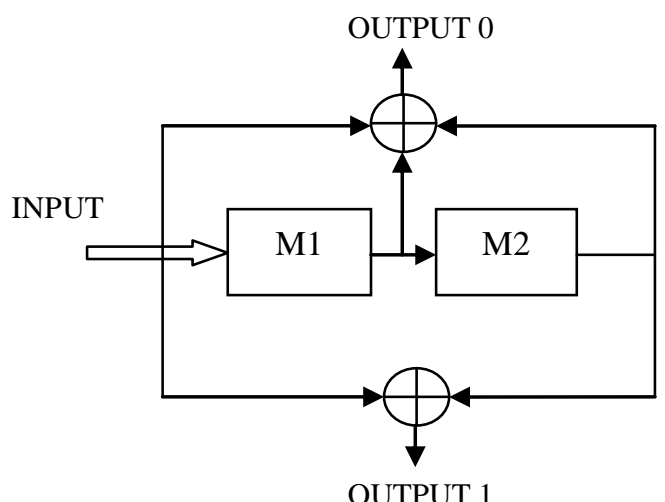

Fig 1. Convolution Encoder; code rate=1/2, $\mathrm{m}=2$

Viterbi decoding is used for decoding the encoded sequence at the receiver side of the WiMAX physical layer. To describe a convolution code, one need to characterize the encoding function $(\mathrm{m})$, so that given an input sequence $\mathrm{m}$, one can readily compute the output sequence $U$.

\subsection{Interleaving}

Interleaving is used to protect the transmission against long sequences of consecutive errors, which are very difficult to correct. These long sequences of error may affect a lot of bits in a row and can then cause many transmitted burst losses. Interleaving, by including some diversity, can facilitate error correction. The encoded data bits are interleaved by a block inter-leaver with a block size corresponding to the number of coded bits per allocated sub channels per OFDM symbol ${ }^{[1]}$.

\subsection{Modulation}

A modulator performs the function of mapping of digital information into analog form in order to transmit the data over the channel which can be done by changing the amplitude, frequency or phase. Various digital modulation techniques can be used are M-PSK and M-QAM [7]. Inverse process, called demodulation, is done by the receiver to recover the transmitted digital information.

\subsection{Pilot Insertion}

The pilot subcarriers are used for various estimation and synchronization purposes.

\subsection{Inverse Fast Fourier Transform (IFFT)}

It converts the frequency domain data to time domain data as the channel is in time domain. IFFT is useful for OFDM because it generates samples of a waveform with frequency component satisfying orthogonality condition.

Similarly FFT converts the time domain to frequency domain as basically we have to work in frequency domain [8]. By calculating the outputs simultaneously and taking advantage of the cyclic properties of the multipliers Fast Fourier Transform (FFT) techniques reduce the number of computations to the order of $N \log N$. The FFT is most efficient when $N$ is a power of two.

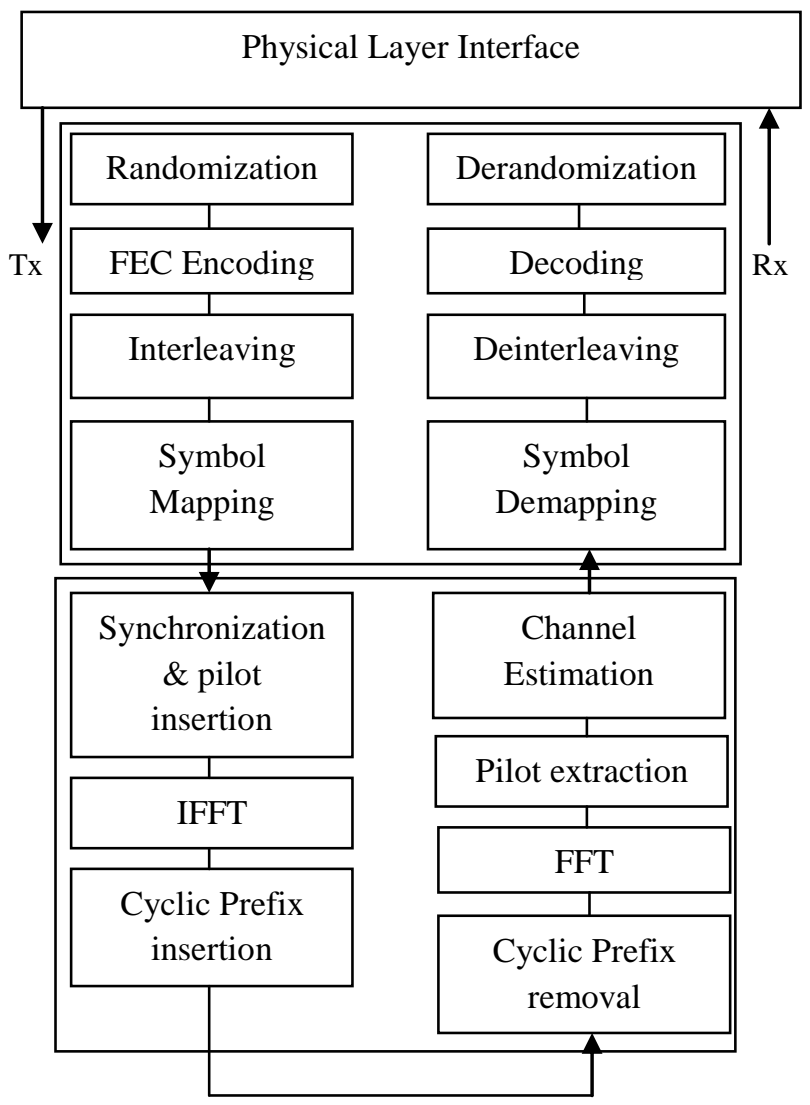

Fig 2. WiMAX Model for Physical Layer (802.16)

\subsection{Cyclic Prefix}

To reduce the ISI, each OFDM symbol is appended with a cyclic prefix (guard interval) [9]. Cyclic prefix includes some of the last data bits added to the starting of the data to ensure no interference within symbols. The length of the prefix is chosen such that it 'absorbs' most of the ISI. The receiver can ignore the signal during the time corresponding to the cyclic prefix; hence the part of the signal used for decoding the data will be virtually ISI-free. The result is little waste of time and power to achieve an almost ISI free channel. The cyclic prefix can be $1 / 4,1 / 8,1 / 16$ or 1/32 of the (useful) symbol length.

\begin{tabular}{|l|c|}
$\longleftrightarrow$ & $\mathrm{N}$ \\
\hline $\mathrm{CP}$ & DATA \\
\end{tabular}

\section{Fig 3. Cyclic Prefix}

\subsection{Communication Channels}

Channels are divided into fast and slow fading channels. A channel is said to be fast if the channel variation is fast compared to the baseband information signal whereas a channel is said to be slow if the channel varies slowly compared to baseband information signal. 
In this paper Rayleigh channel is taken into consideration for the analysis purpose. Constructive and destructive nature of multipath components in flat fading channels can be approximated by using Rayleigh distribution if there is no line of sight which means when there is no direct path between the transmitter and receiver. The received signal can be simplified to:

$$
r(t)=s(t) * h(t)+n(t)
$$

where $\mathrm{h}(\mathrm{t})$ is the random channel matrix having Rayleigh distribution and $n(t)$ is the additive white Gaussian noise. The Rayleigh distribution is basically the magnitude of the sum of two equal independent orthogonal Gaussian random variables.

\subsection{Receiver Side}

At the receiving side, a reverse process (including deinterleaving and decoding) is executed to obtain the original data bits. As the deinterleaving process only changes the order of received data, the error probability is intact [9].

\section{MULTI INPUT MULTI OUTPUT (MIMO) SYSTEMS}

Multiple-input and multiple-output or MIMO, refers to the use of multiple antennas both at the transmitter and receiver to improve the performance of radio communication systems. MIMO technology has attracted attention in wireless communications, since it offers significant increases in data throughput and link range without additional bandwidth or transmit power. It achieves this by higher spectral efficiency and link reliability or diversity (reduced fading) [10]

\section{Transmitting}

Antennas

MIMO Channel

Receiving

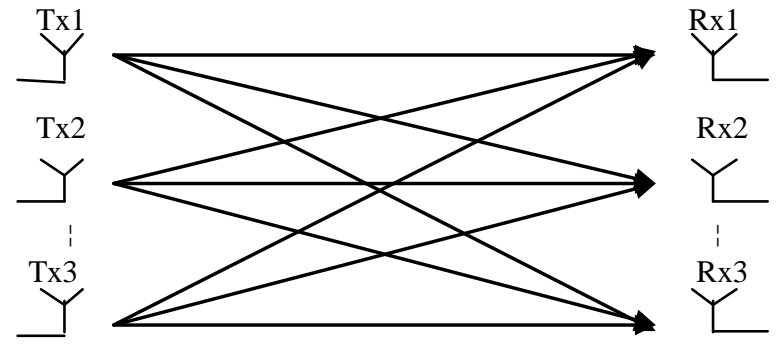

Fig 4. Block Diagram of MIMO system with M Transmitters and $\mathbf{N}$ Receivers

\section{MIMO TECHNIQUES}

MIMO can be sub-divided into three main categories, spatial multiplexing or SM, diversity coding and beamforming.

\subsection{Spatial Diversity}

Diversity coding techniques are used when there is no channel knowledge at the transmitter. In diversity methods a single stream (unlike multiple streams in spatial multiplexing) is transmitted, but the signal is a coded using technique called space-time coding [11]. The signal is emitted from each of the transmit antennas using certain principles of full or near orthogonal coding. Diversity exploits the independent fading in the multiple antenna links to enhance signal diversity.

MIMO take advantage of the spatial diversity obtained by placing separate antennas in a dense multipath scattering environment. These systems can be implemented in a number of different ways to obtain either a diversity gain to combat signal fading with the help of spatial diversity or a capacity improvement with the help of spatial multiplexing techniques of MIMO systems. Therefore, the receiver can reliably decode the transmitted signal using these received signals. This technique involves Space Time Block Coding (STBC) and Space Time Trellis Coding (STTC). Diversity techniques can be implemented into different ways [12].

\subsection{Spatial Multiplexing}

Spatial multiplexing requires MIMO antenna configuration. In spatial multiplexing, a signal is divided into different streams and each stream is transmitted from a different transmit antenna in the same frequency channel. If these signals arrive at the receiver antenna array with sufficiently different spatial signatures, the receiver can separate these streams, creating parallel channels for free. Spatial multiplexing is very powerful technique for increasing channel capacity at higher Signal to Noise Ratio (SNR). It can be used with or without transmit channel knowledge. This technique includes V-BLAST technology [13]

\subsection{Beamforming}

This technique involves directing the signal in the desired direction so that the signal going in the desired direction is increased and signal going in the wrong direction is reduced. In beamforming, the same signal is emitted from each of the transmit antennas with appropriate phase (and sometimes gain) weighting such that the signal power is maximized at the receiver input. Beamforming mainly increase the signal gain from constructive combining and to reduce the multipath fading effect [14]. In the absence of scattering, beamforming results in a well defined directional pattern.

\section{WiMAX-MIMO SYSTEMS}

WiMAX-MIMO systems are mainly implemented to get the benefits of both WiMAX as well as the MIMO systems in order to provide high speed broadband internet access to the users [15]. Main motive of combining WiMAX and Spatial Diversity technique of MIMO systems is to higher data rates by lowering down the BER and SNR of the system. The performance of simple WiMAX physical layer can be enhanced by employing MIMO techniques in it such that diversity gain or capacity gain can be achieved. The block diagram of proposed WiMAX-MIMO systems is given in Figure 5 .

This paper mainly focuses upon the spatial diversity technique of MIMO systems so that diversity gain can be obtained by transmitting same information through different individual antennas present at the transmitter side of MIMO systems such that the received signal is the coherent combination of all the transmitted signals. 


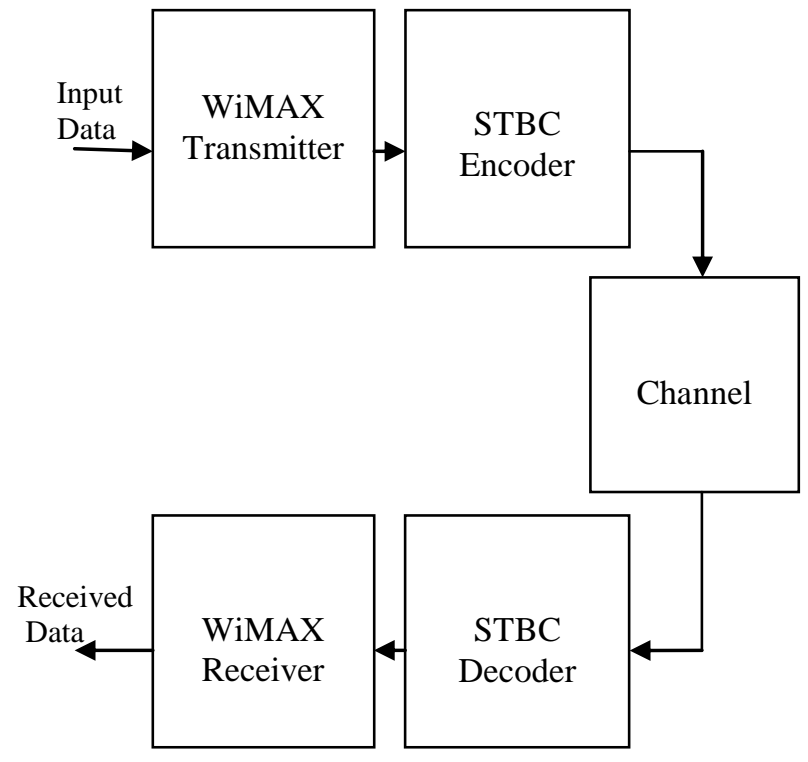

Fig 5. WiMAX-MIMO System

Combination of both the WiMAX and MIMO technology provides an efficient solution for high data rate transmission. This system gives better BER performance in comparison to simple WiMAX without MIMO. Spatial diversity is obtained by placing separate antennas in a dense multipath scattering environment. This can be done in many ways to obtain diversity gain to combat signal fading. STBC Encoder and Decoder are employed at the transmitter and receiver side of the WiMAX PHY layer before and after the channel respectively.

This paper analyze the spatial diversity technique of MIMO systems combined with WiMAX in order to achieve higher data rates by lowering the Bit Error Rate of the system to achieve better performance and results. Results are obtained for Rayleigh channel.

\section{RESULTS AND SIMULATIONS}

This paper analyzes the behavior of WiMAX-MIMO systems under different modulation schemes with different code rates for Rayleigh channel. Results are shown in the form of SNR vs BER plot for different modulations. The simulations are done for STBC 3X3 i.e. 3 antennas at the transmitter and the receiver side so as to attain more efficient performance of the WiMAX-MIMO systems as compared to simple WiMAX physical layer simulation.

In order to analyze the WiMAX-MIMO system using STBC 3X3 technique, firstly WiMAX physical layer has been simulated with its each and every block and afterwards it is combined with the STBC 3X3 technique of MIMO systems using different lower and higher modulation schemes under different code rates that enhances the performance of the system.

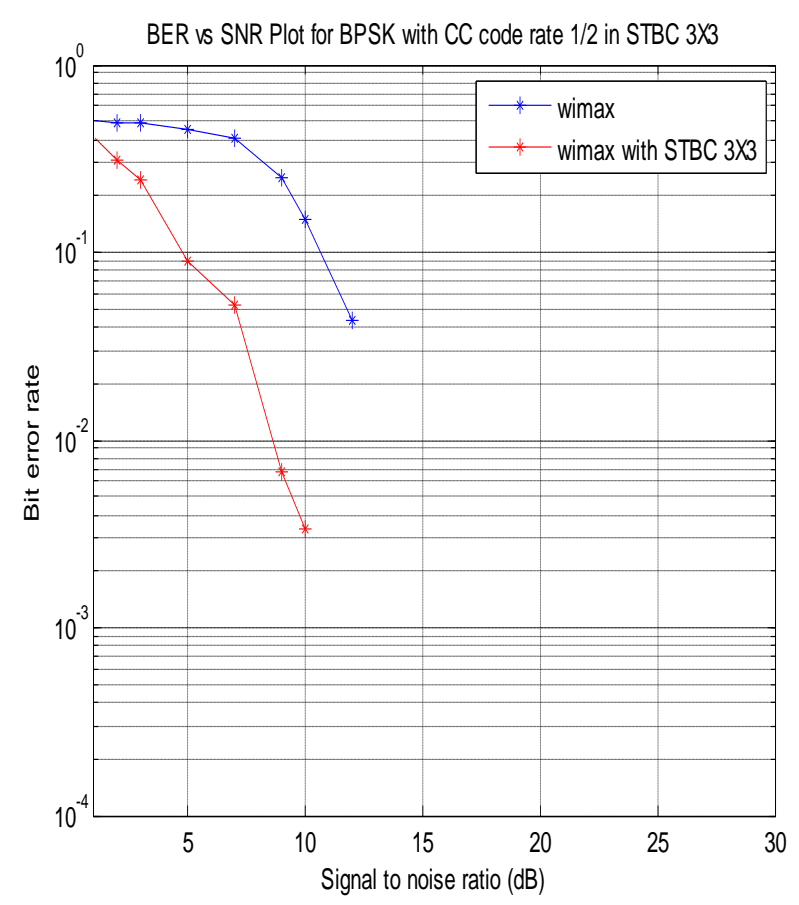

(a) BPSK with CC code rate $1 / 2$

In this graph we are able to get $2 \mathrm{~dB}$ improvement in SNR is shown when we employ Spatial Diversity technique of MIMO in WiMAX in the presence of Rayleigh channel.

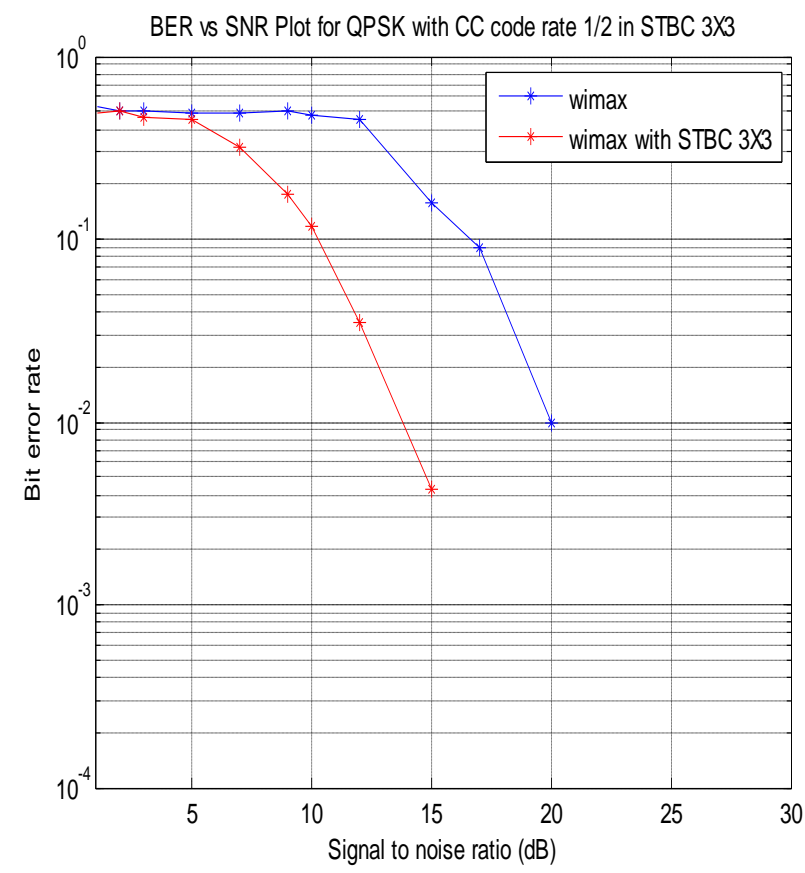

(b) QPSK with CC code rate $1 / 2$

QPSK modulation with $\mathrm{CC}$ code rate $1 / 2$ gives $5 \mathrm{~dB}$ improvement in SNR when combining WiMAX with MIMO Spatial Diversity technique. Rayleigh channel is used for the analysis. 


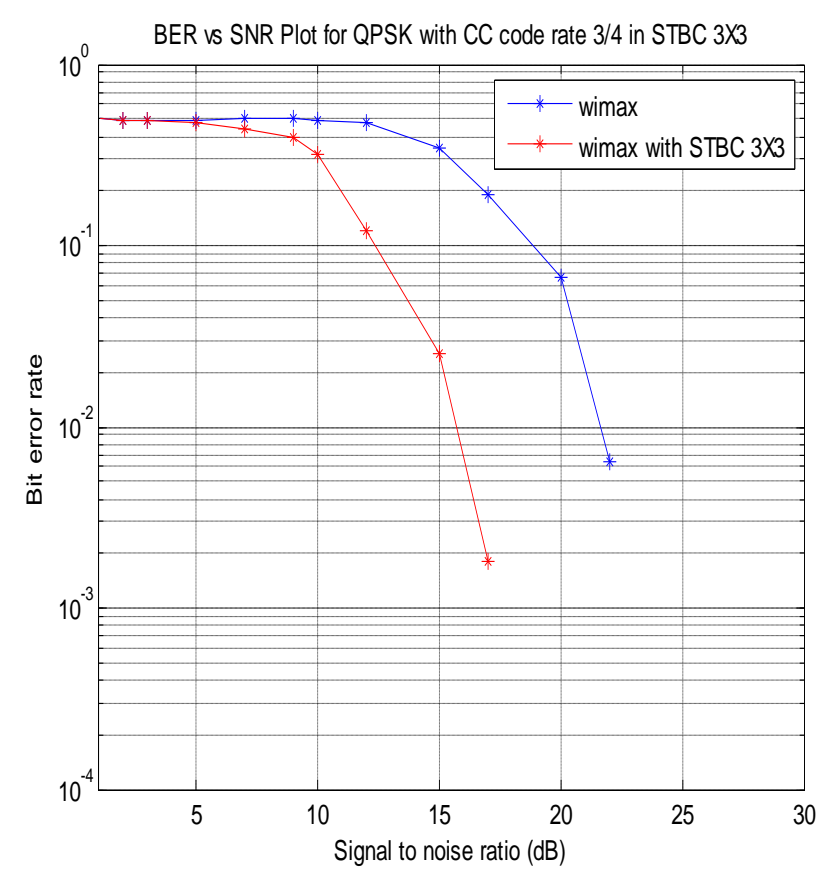

(c) QPSK with CC code rate $3 / 4$

SNR improvement of $5 \mathrm{~dB}$ can be seen when we use QPSK modulation with CC code rate of $3 / 4$ in WiMAX-MIMO system compared to simple WiMAX using Rayleigh channel.

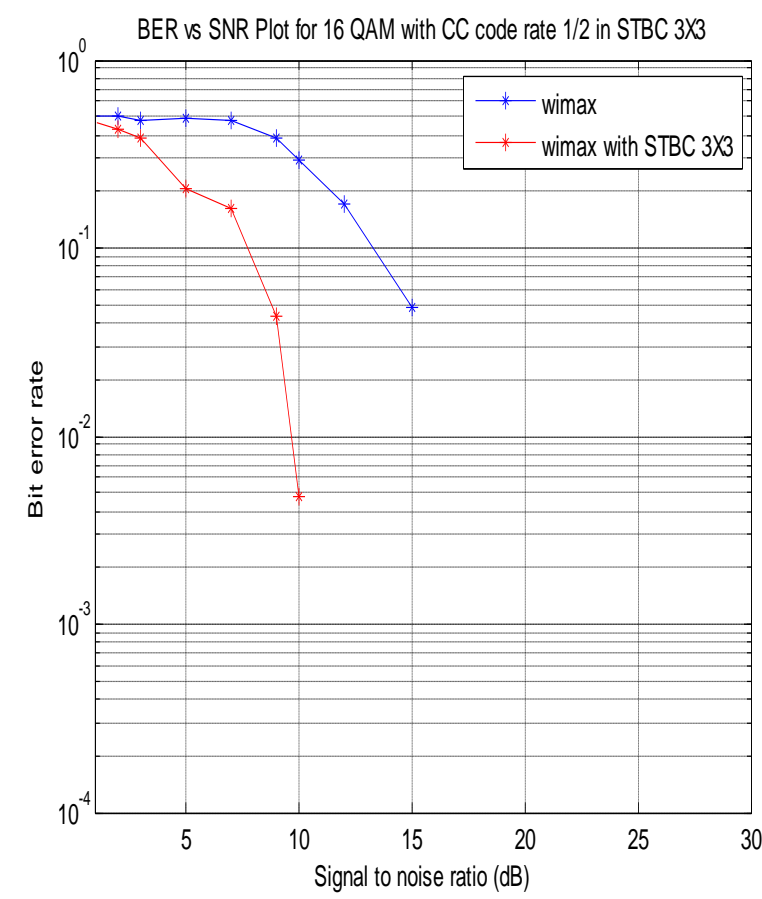

(d) 16QAM with $\mathrm{CC}$ code rate $1 / 2$

As seen from the graph, SNR improvement of $5 \mathrm{~dB}$ is there using WiMAX with Spatial Diversity technique of MIMO technology in the presence of Rayleigh channel.

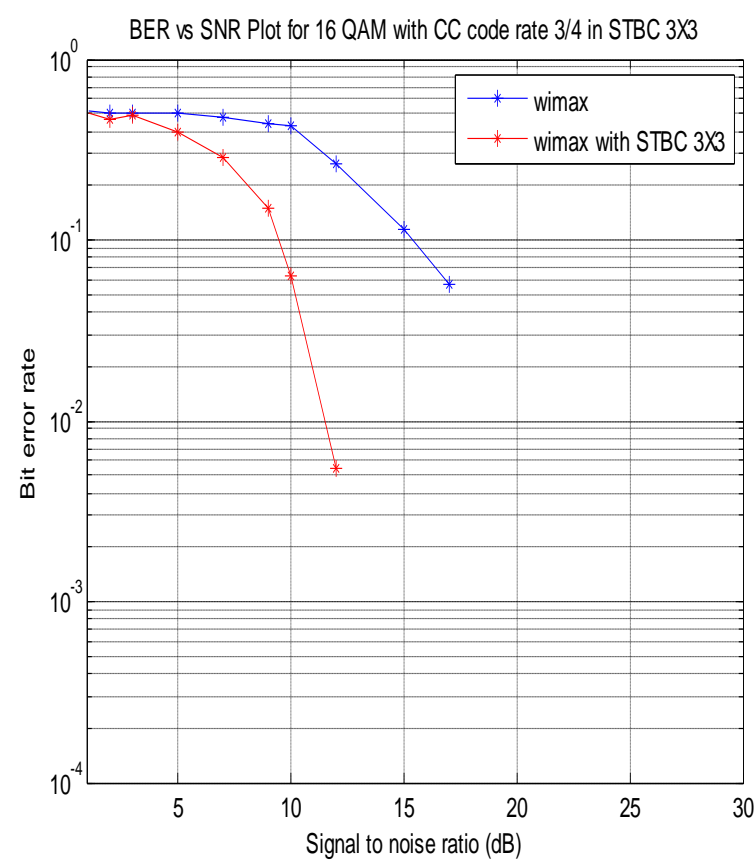

(e) 16 QAM with $\mathrm{CC}$ code rate $3 / 4$

Again as we can see there is an improvement of $5 \mathrm{~dB}$ in SNR using Rayleigh channel with combined effect of WiMAXMIMO technology.

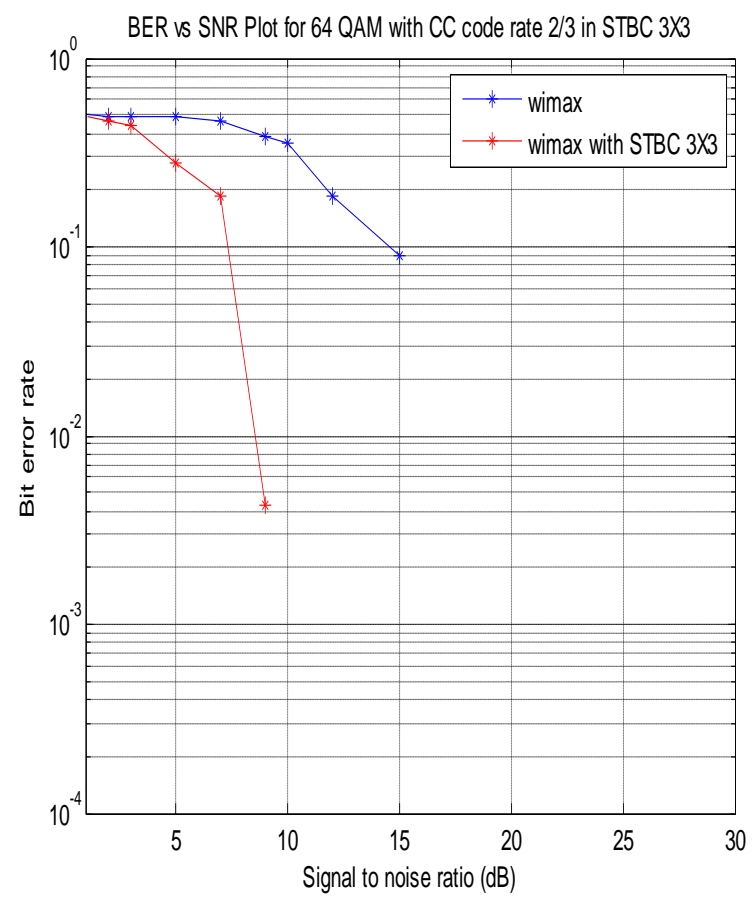

(f) 64 QAM with $\mathrm{CC}$ code rate $2 / 3$

SNR improvement of 6dB incase of 64QAM with CC code rate $2 / 3$ can be seen in when we use WiMAX-MIMO in the presence of Rayleigh channel. 


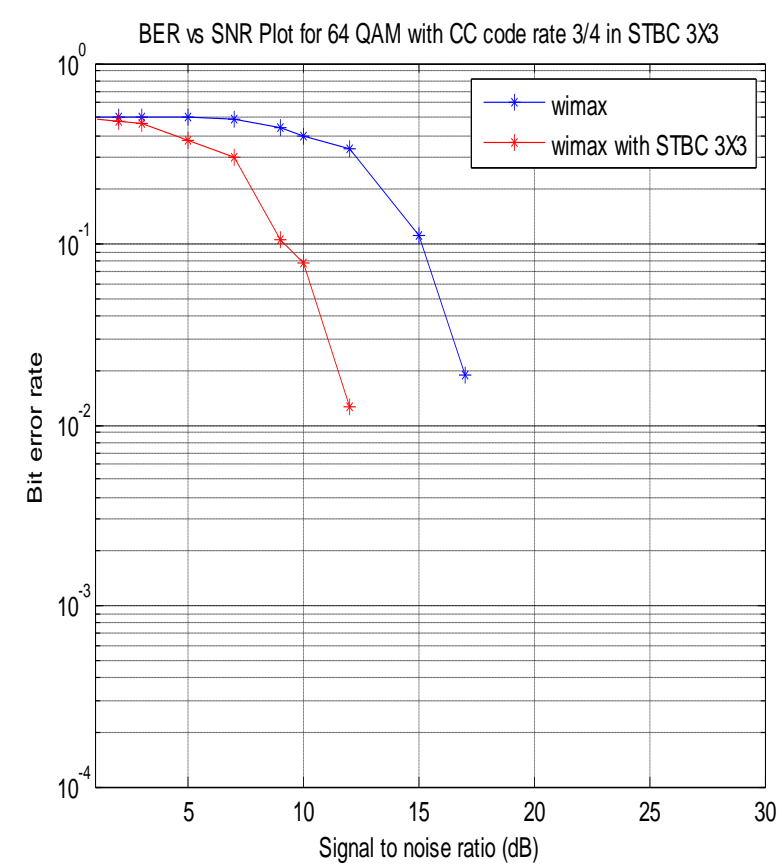

(g) 64 QAM with CC code rate 3/4

This graph shows an SNR improvement of $5 \mathrm{~dB}$ using WiMAX-MIMO system in the presence of Rayleigh channel.

Fig 6. BER vs SNR plots for Rayleigh channel a) BPSK code rate $1 / 2$ b) QPSK code rate $1 / 2$ c) QPSK code rate 3/4 d) 16 QAM code rate $1 / 2$ e) 16 QAM code rate $3 / 4$ (f) 64 QAM code rate $2 / 3$ (g) 64 QAM code rate $3 / 4$

The performance in the form of BER vs SNR plots for different modulations over Rayleigh channel for WiMAXMIMO system with different CC code rates have been presented in Figure 6 (a)-(g). Each graph shows an improvement in SNR using spatial diversity technique of MIMO system which is given in the following table.

Table 1: SNR improvement in Rayleigh channel by using Spatial Diversity in WiMAX

\begin{tabular}{|c|c|}
\hline MODULATION & $\begin{array}{c}\text { SNR Improvement } \\
\text { using Rayleigh } \\
\text { channel }(\mathrm{dB})\end{array}$ \\
\hline BPSK code rate $1 / 2$ & $2 \mathrm{~dB}$ \\
\hline QPSK code rate $1 / 2$ & $5 \mathrm{~dB}$ \\
\hline QPSK code rate $3 / 4$ & $5 \mathrm{~dB}$ \\
\hline 16 QAM code rate $1 / 2$ & $5 \mathrm{~dB}$ \\
\hline 16 QAM code rate 3/4 & $5 \mathrm{~dB}$ \\
\hline 64QAM code rate $2 / 3$ & $6 \mathrm{~dB}$ \\
\hline 64QAM code rate 3/4 & $5 \mathrm{~dB}$ \\
\hline
\end{tabular}

\section{CONCLUSION}

In this paper effect of employing MIMO spatial diversity in 802.16e PHY layer has been simulated. Rayleigh channel has been taken into account for the analysis purpose. Simulations show that there is improvement in the SNR value as well as the Bit Error Rate. Results have shown that BER reduces when we employ MIMO system in WiMAX in comparison to simple WiMAX. This shows that employing MIMO in WiMAX improves the performance of the system

\section{ACKNOWLEDMENTS}

Foremost, I would like to express my sincere gratitude to $\mathbf{M r}$. Lavish Kansal who gave his full support in the compilation of this report with his stimulating suggestions and encouragement to go ahead in all the time of the thesis. I would also like to thank Ms. Navpreet Kaur for her kind support during this work. I am also thankful to Dr. Sandeep Chakraborty, Head, Electronics and Communication Engineering Department, for providing us with the adequate infrastructure in carrying more interesting the work. At last but not the least my gratitude towards my parents, I would also like to thank God for the strength that keep me standing and for the hope that keep me believing that this report would be possible.

\section{REFERENCES}

[1] IEEE standard for Local and Metropolitan Area NetworksPart 16: Air interface for Fixed Broadband Wireless Access Systems, IEEE Std. 802.16, Oct. 2004.

[2] IEEE standard 802.16-2005, IEEE standard for Local and Metropolitan Area Networks-Part16: Air Interface for Fixed and Mobile Broadband wireless Access system, Feb 2006.

[3] IEEE 802.16 WG,"IEEE Standard for Local and Metropolitan Area Network Part 16: Air Interface for Fixed Broadband Wireless Access Systems" IEEE Std 802.16-2004 p.1 - p.857

[4] IEEE 802.16WG,"IEEE standard for local and metropolitan area networks part 16: Air interface for fixed and mobile broadband wireless access systems, Amendment 2," IEEE 802.16 Standard, December 2005.

[5] V. Erceg, K.V.S. Hari, M.S. Smith and D.S. Baum "Channel Models for Fixed Wireless Applications", IEEE 802.16.3 Task Group Contributions, February 2001.

[6] B. Muquet, E. Biglieri, A. Goldsmith and H. Sari, "MIMO Techniques for Mobile WiMAX Systems", SEQUANS Communications White Paper, pp 1-18, 2006.

[7] M. N. Khan and S. Ghuari, "The WiMAX 802.16e Physical Layer Model", International Conference on Wireless, Mobile and Multimedia Networks, pp 117-120, 2007.

[8] S. Bansal, R. Upadhyay, "Performance Improvement of Wi-Max IEEE 802.16e in Presence of Different FEC Codes", First International Conference on Computational Intelligence, Communication Systems and Networks, IEEE Computer Society, pp 226-229, 2009.

[9] M. Wang, "WiMAX Physical Layer: Specifications Overview and Performance Evaluation", 2nd IEEE CCNC Research Student Workshop, pp 10-12, 2011.

[10] A. Sadat and W. B. Mikhael, "Fast Fourier Transform for High Speed OFDM Wireless Multimedia System", 44th IEEE Midwest symposium on circuits and systems, Vol. 2, pp 938-942, 2001 
[11] A. Vahlin and N. Holte, "Use of guard interval in OFDM in multipath channels", Electronics Letter, Vol. 30, Issue 24, pp 2015-2016, 1996.

[12] S. Alamouti, "A simple transmit diversity technique for wireless communications", IEEE Journal on Selected Areas of Communication, Vol. 16, pp 1451-1458, Oct. 1998.

[13] H. Hourani, "An overview of diversity techniques in wireless communication systems," IEEE JSAC , pp 1200-5, October 2004.

[14] V. Tarokh, H. Jafarkhani and A. R. Calderbank, "Spacetime block codes from orthogonal designs", IEEE Transactions on Information Theory, Vol. 45, pp 14561467, July 1999.
[15] P. W. Wolniansky, G. J. Foschini, G. D. Golden and R. A. Valenzuela, "V-Blast: An architecture for realizing very high data rates over the rich-scattering channel", International Symposium on Signals, Systems and Electronics, pp 295-300, 1998.

[16] J. Huang, J. Zhang, Z. Liu, J. Li and X. Li, "Transmit Beamforming for MIMO-OFDM Systems with Limited Feedback", IEEE Vehicular Technology Conference, pp $1-5,2008$.

[17] O. Arafat, K. Dimyati, "Performance Parameter of Mobile WiMAX: A Study on the Physical Layer of Mobile WiMAX under Different Communication Channels \& Modulation Technique", Second International Conference on Computer Engineering and Applications, pp 533-537, 2010. 